\title{
Remote payload transportation using an aircraft-towed flexible cable system
}

\author{
D. E. Sgarioto* P. Williams P. M. Trivailo
}

(Received 27 October 2005; revised 3 August 2006)

\begin{abstract}
We demonstrate the concept of using a cable towed beneath an aircraft to transport payloads without landing. The cable is modelled using a lumped parameter approach and the equations of motion are derived using Kane's method. Full three-dimensional dynamic coupling is preserved between the aircraft dynamics and the motion of the cable and payload. The system is controlled via aircraft maneuvering and prescribing the reel acceleration of the cable. A simulated payload transportation operation is numerically represented as a nonlinear optimal control problem and solved using a direct transcription technique implemented in Matlab. Numerical results are presented for a typical payload transportation operation.
\end{abstract}

*School of Aerospace, Mechanical \& Manufacturing Engineering, RMIT University, Melbourne, AustraliA. mailto:S9908712@student.rmit.edu.au

See http://anziamj.austms.org.au/V47EMAC2005/Sgarioto for this article, (c) Austral. Mathematical Soc. 2006. Published September 11, 2006. ISSN 1446-8735 


\section{Contents}

1 Introduction

C232

2 Aerial towed-cable system modelling

C233

3 Control system development

C237

4 Results

C240

5 Conclusions

$\mathrm{C} 242$

References

C243

\section{Introduction}

Many applications have been proposed for aerial towed-cable systems, including the snatch pick-up of payloads [1], precision water-bombing and remote payload transportation $[2,3,4,5]$. For these to be successful, accurate guidance of the cable tip is crucial, yet most prior work in this area has not focused on this. Of the few studies that have dealt specifically with controlling the motion of the cable tip, Jun et al. [1] employed Linear Quadratic Regulator control to actively control the aerodynamic surfaces of the payload to "hit" a stationary ground target. Trivailo et al. [2] used a simple model of the cable (straight, inelastic) to formulate an optimal control problem for rendezvousing the cable tip with ground targets. Sgarioto and Trivailo [3] extended this work by using a dual rigid-link cable model and incorporated aircraft maneuvers into the control formulation. More recently, Williams et al. [4] used a rigid multi-link cable model to study rendezvous problems having multiple ground targets separated by elevated terrain. Williams et al. [5] used the concept of differential flatness to study multiple rendezvous problems with aircraft dynamic coupling using constrained optimization strategies. 

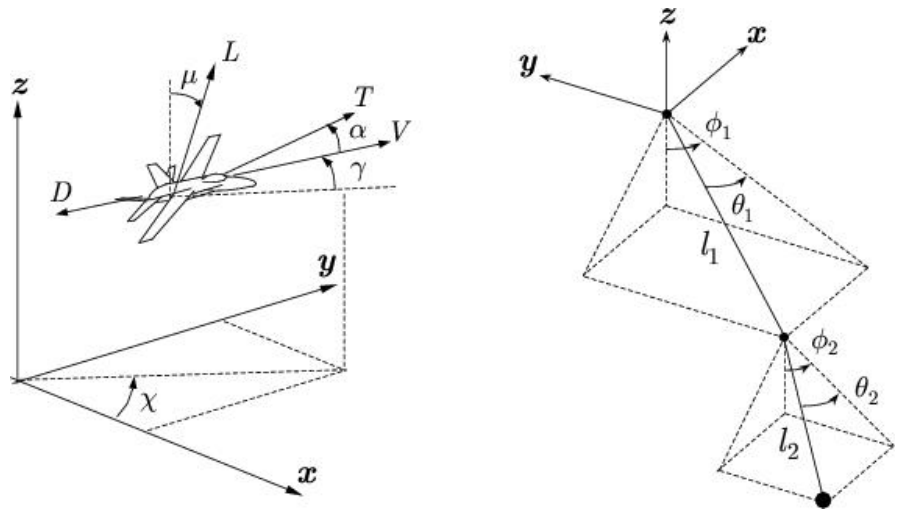

FiguRE 1: Aircraft towed-cable system model

Our aim is to investigate the concept of remote transportation of payloads using a cable towed beneath an aircraft. A guidance algorithm designed using nonlinear optimal control is employed to control the tip of the cable. This work extends previous research by considering the entire three dimensional motion of the system, including the full dynamic coupling between the aircraft and the cable. Aircraft maneuvers are considered in the control methodology and a terrain avoidance capability will be expanded into three dimensions.

\section{Aerial towed-cable system modelling}

The model of the aircraft and towed cable that constitute the aerial towedcable system is depicted in Figure 1. The equations of motion for the dynamically coupled aerial towed-cable system are derived using Kane's Equations. The motion of the system is characterized by the following variables: the inplane $\left(\theta_{1}, \theta_{2}\right)$ and out-of-plane $\left(\phi_{1}, \phi_{2}\right)$ cable orientation angles; the position of the aircraft $(x, y, z)$; the upper cable segment length and length rate $\left(l_{1}, \dot{l}_{1}\right)$; the in-plane $\left(\dot{\theta}_{1}, \dot{\theta}_{2}\right)$ and out-of-plane $\left(\dot{\phi}_{1}, \dot{\phi}_{2}\right)$ cable angular rates; the aircraft 
velocity, flight path and heading angles $(V, \gamma, \chi)$ and the aircraft angle of attack $\alpha$ and velocity roll angle $\mu$. The actuators chosen to control the system are the reel acceleration of the cable $\ddot{l}_{1}$, the engine throttle $\eta$, the rate of change of the aircraft angle of attack $\dot{\alpha}$ and velocity roll angle $\dot{\mu}$.

Essentially, the aircraft is modelled as a point mass, assuming that the rigid body rotations of the aircraft are much faster than its translational motion. It is assumed that the aircraft is prevented from side-slipping and that the engine thrust is aligned with the aircraft longitudinal body axis. The aircraft tows a homogeneous sphere of constant mass, drag coefficient and diameter via a rigid massive cable, discretized using two rigid links of equal diameter and similar material. The cable is modelled using a lumped parameter approach, which concentrates the inertial and external forces at point masses articulated by frictionless hinges. The cable is capable of deployment and retrieval by prescribing the reel acceleration of the first segment only; the lower link is fixed in length. The equations of motion for the system are now derived. Define the aircraft inertial position by

$$
\mathbf{r}_{0}=x \mathbf{i}+y \mathbf{j}+z \mathbf{k} .
$$

The position of the first segment end $\left(\mathbf{r}_{1}\right)$ and cable tip $\left(\mathbf{r}_{2}\right)$ are

$$
\begin{aligned}
& \mathbf{r}_{1}=\mathbf{r}_{0}-l_{1} \sin \theta_{1} \mathbf{i}-l_{1} \cos \theta_{1} \sin \phi_{1} \mathbf{j}-l_{1} \cos \theta_{1} \cos \phi_{1} \mathbf{k} \\
& \mathbf{r}_{2}=\mathbf{r}_{1}-l_{2} \sin \theta_{2} \mathbf{i}-l_{2} \cos \theta_{2} \sin \phi_{2} \mathbf{j}-l_{2} \cos \theta_{2} \cos \phi_{2} \mathbf{k}
\end{aligned}
$$

Differentiating Equations (1)-(3) with respect to time yields the inertial velocity of the aircraft and each cable segment end:

$$
\begin{aligned}
\mathbf{v}_{0}= & \dot{x} \mathbf{i}+\dot{y} \mathbf{j}+\dot{z} \mathbf{k}, \\
\mathbf{v}_{1}= & \mathbf{v}_{0}+\left(-\dot{l}_{1} \sin \theta_{1}-l_{1} \dot{\theta}_{1} \cos \theta_{1}\right) \mathbf{i} \\
& -\left(\dot{l}_{1} \cos \theta_{1} \sin \phi_{1}-l_{1} \dot{\theta}_{1} \sin \theta_{1} \sin \phi_{1}+l_{1} \dot{\phi}_{1} \cos \theta_{1} \cos \phi_{1}\right) \mathbf{j} \\
& -\left(\dot{l}_{1} \cos \phi_{1} \cos \theta_{1}-l_{1} \dot{\phi}_{1} \sin \phi_{1} \cos \theta_{1}-l_{1} \dot{\theta}_{1} \cos \phi_{1} \sin \theta_{1}\right) \mathbf{k},
\end{aligned}
$$




$$
\begin{aligned}
\mathbf{v}_{2}= & \mathbf{v}_{1}-\left(l_{2} \dot{\theta}_{2} \cos \theta_{2}\right) \mathbf{i} \\
& +\left(l_{2} \dot{\theta}_{2} \sin \theta_{2} \sin \phi_{2}-l_{2} \dot{\phi}_{2} \cos \theta_{2} \cos \phi_{2}\right) \mathbf{j} \\
& +\left(l_{2} \dot{\phi}_{2} \sin \phi_{2} \cos \theta_{2}+l_{2} \dot{\theta}_{2} \cos \phi_{2} \sin \theta_{2}\right) \mathbf{k} .
\end{aligned}
$$

The inertial velocity of the aircraft expressed in velocity coordinates is

$$
\begin{aligned}
\dot{x} & =V \cos \gamma \cos \chi, \\
\dot{y} & =V \cos \gamma \sin \chi, \\
\dot{z} & =V \sin \gamma .
\end{aligned}
$$

Differentiating Equations (7)-(9) yields the inertial acceleration of the aircraft:

$$
\begin{aligned}
& \ddot{x}=\dot{V} \cos \gamma \cos \chi-V \dot{\gamma} \sin \gamma \cos \chi-V \dot{\chi} \sin \chi \cos \gamma, \\
& \ddot{y}=\dot{V} \cos \gamma \sin \chi-V \dot{\gamma} \sin \gamma \sin \chi+V \dot{\chi} \cos \chi \cos \gamma, \\
& \ddot{z}=\dot{V} \sin \gamma+V \dot{\gamma} \cos \gamma .
\end{aligned}
$$

The aircraft equations of motion are expressed in velocity coordinates as

$$
\begin{aligned}
m_{0} \dot{V} & =T \cos \alpha-D-m_{0} g \sin \gamma, \\
m_{0} V \dot{\gamma} & =(L+T \sin \alpha) \cos \mu-m_{0} g \cos \gamma, \\
m_{0} V \dot{\chi} & =(L+T \sin \alpha) \sin \mu,
\end{aligned}
$$

where $m_{0}$ is the mass of the aircraft, $L$ and $D$ are the lift and drag acting on the aircraft, whereas the engine thrust of the aircraft is

$$
T=T_{\max } \eta
$$

The equations of motion for the aircraft expressed in the inertial coordinate system are found once Equations (10)-(12) are inverted and $(\dot{V}, \dot{\gamma}, \dot{\chi})$ are determined. The result is then substituted into Equations (13)-(15) to yield the required aircraft dynamical motion equations:

$$
m_{0} \ddot{x}=\left(T \cos \alpha-D-m_{0} g \sin \gamma\right) \cos \gamma \cos \chi-[(L+T \sin \alpha) \sin \mu] \sin \chi
$$




$$
-\left[(L+T \sin \alpha) \cos \mu-m_{0} g \cos \gamma\right] \sin \gamma \cos \chi,
$$

$$
\begin{aligned}
m_{0} \ddot{y}= & \left(T \cos \alpha-D-m_{0} g \sin \gamma\right) \sin \gamma \sin \chi+[(L+T \sin \alpha) \sin \mu] \cos \chi \\
& -\left[(L+T \sin \alpha) \cos \mu-m_{0} g \cos \gamma\right] \sin \gamma \sin \chi \\
m_{0} \ddot{z}= & \left(T \cos \alpha-D-m_{0} g \sin \gamma\right) \sin \gamma \\
& +\left[(L+T \sin \alpha) \cos \mu-m_{0} g \cos \gamma\right] \cos \gamma .
\end{aligned}
$$

The standard form of Kane's Equations is

$$
F^{\star}+F=0,
$$

where the generalised inertia and active forces, $F^{\star}$ and $F$ respectively, are

$$
\begin{aligned}
F^{\star} & =\sum_{j=1}^{3} m_{j} \mathbf{a}_{j} \cdot \frac{\partial \mathbf{v}_{j}}{\partial \dot{q}_{k}}, \quad k=1,2, \ldots, 7, \\
F & =\sum_{j=1}^{3} \mathbf{F}_{j} \cdot \frac{\partial \mathbf{v}_{j}}{\partial \dot{q}_{k}}, \quad k=1,2, \ldots, 7 .
\end{aligned}
$$

where $\mathbf{a}_{j}$ is the acceleration vector obtained by differentiating Equations (4)(6) with respect to time, and $\mathbf{F}_{j}$ are the external forces acting on the system. The mass vector

$$
m=\left[\begin{array}{lll}
m_{0} & \frac{1}{8} \pi \rho_{c}\left(l_{1}+l_{2}\right) d^{2} & \frac{1}{8} \pi \rho_{c}\left(l_{1}+l_{2}\right) d^{2}+m_{p}
\end{array}\right],
$$

where $d$ is the cable diameter and $m_{p}$ is the payload mass. The vector $\dot{q}$ in Equations (21) and (22) is

$$
\dot{q}=\left[\begin{array}{lllllll}
\dot{x} & \dot{y} & \dot{z} & \dot{\theta}_{1} & \dot{\phi}_{1} & \dot{\theta}_{2} & \dot{\phi}_{2}
\end{array}\right],
$$

where $(\dot{x}, \dot{y}, \dot{z})$ are given by Equations (7)-(9).

The predominant external forces acting on the system are gravitational and aerodynamic. Gravity is assumed to be invariant with altitude, although the atmospheric air density is not and standard conditions apply. The aerodynamic forces acting on the aircraft are well known [5]. With respect to 
aerodynamic forces acting on the cable, each cable segment is treated as an inclined cylinder. The aerodynamic forces acting on the cable are calculated by determining the force on each cable segment, averaging these values, then lumping this to each mass element. The aerodynamic and gravitational forces acting on the payload are lumped at the cable tip. Williams et al. [5] give the full treatment of the external forces acting on the cable. Application of Kane's Equations to the system yields the equations of motion

$$
\ddot{q}=[M] B,
$$

where $[M]$ is the mass matrix of the system, and $B$ is the vector making up the external and control forces acting on the system. The equations of motion are then cast in state-space form by defining

$$
\dot{\zeta}=\left[\begin{array}{llllll}
\dot{q} & \ddot{q} & \dot{l}_{1} & \ddot{l}_{1} & \dot{\alpha} & \dot{\mu}
\end{array}\right]^{T} .
$$

Hence the aircraft towed-cable system can be represented by eighteen state equations of motion, with significant coupling existing between the acceleration of the aircraft and the dynamics of the cable/payload combination.

\section{Control system development}

The control scenario considered in this paper constitutes the guidance problem depicted in Figure 2. The aircraft is flown and the cable deployed so that the cable tip moves from an initial state to instantaneously rendezvous with a known target, while avoiding collision with the terrain. The scenario begins with the system in equilibrium and ends once rendezvous takes place. To produce a unique trajectory with smooth reel and aircraft dynamics, the guidance problem is solved so as to minimize the reel acceleration $\ddot{l}_{1}$ and the rate of change of the aircraft angle of attack $\dot{\alpha}$ and velocity roll angle $\dot{\mu}$. 


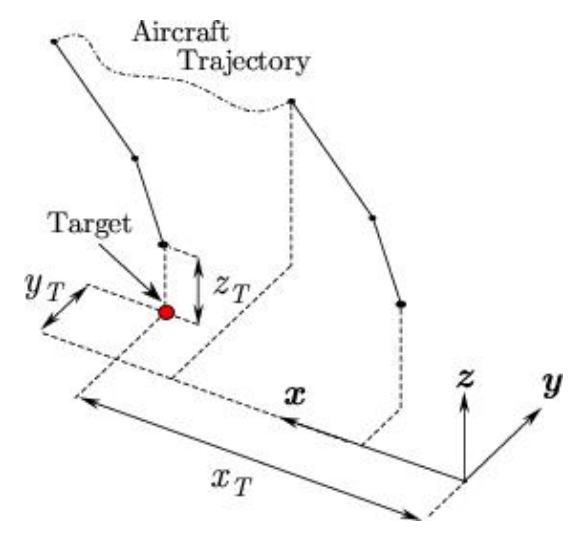

Figure 2: Control scenario: cable tip guidance problem

Mathematically, the optimal control problem is posed as follows. Determine the control input

$$
\mathbf{u}=\left[\begin{array}{llll}
\ddot{l}_{1} & \eta & \dot{\alpha} & \dot{\mu}
\end{array}\right],
$$

and the corresponding state trajectory $\zeta=\left[\begin{array}{llllllllllllllllll}\theta_{1} & \phi_{1} & \theta_{2} & \phi_{2} & x & y & z & l_{1} & \dot{\theta}_{1} & \dot{\phi}_{1} & \dot{\theta}_{2} & \dot{\phi}_{2} & V & \gamma & \chi & \dot{l}_{1} & \alpha & \mu\end{array}\right]$,

to minimize the performance index

$$
J=\frac{1}{2} \int_{t_{0}}^{t_{f}}\left(\ddot{l}_{1}^{2}+\dot{\alpha}^{2}+\dot{\mu}^{2}\right) d t
$$

subject to system dynamical equations of motion

$$
\dot{\zeta}=\mathbf{f}(\zeta(t), \mathbf{u}(t), t)
$$

with boundary conditions

$$
\begin{aligned}
& \psi_{0}\left(\zeta\left(t_{0}\right), t_{0}\right)=\mathbf{0}, \\
& \psi_{f}\left(\zeta\left(t_{f}\right), t_{f}\right)=\mathbf{0},
\end{aligned}
$$


and path constraints

$$
\mathbf{g}(\zeta(t), t) \leq \mathbf{0} .
$$

The chosen initial conditions are the equilibrium configuration of the system for a given tow speed, cable length and position of the aircraft, calculated using a nonlinear root finding procedure. The final conditions at rendezvous are specified in terms of the cable tip:

$$
\begin{aligned}
x\left(t_{f}\right)-\sum_{j=1}^{2} l_{j}\left(t_{f}\right) \sin \theta_{j}\left(t_{f}\right) & =x_{T}, \\
y\left(t_{f}\right)-\sum_{j=1}^{2} l_{j}\left(t_{f}\right) \cos \theta_{j}\left(t_{f}\right) \sin \phi_{j}\left(t_{f}\right) & =y_{T}, \\
z\left(t_{f}\right)-\sum_{j=1}^{2} l_{j}\left(t_{f}\right) \cos \theta_{j}\left(t_{f}\right) \cos \phi_{j}\left(t_{f}\right) & =z_{T},
\end{aligned}
$$

where the coordinates $\left(x_{T}, y_{T}, z_{T}\right)$ are the known location of the ground target. At rendezvous the cable reel rate is constrained to be zero:

$$
\dot{l}_{1}\left(t_{f}\right)=0 .
$$

An explicit path constraint prevents the cable tip from colliding with the ground:

$$
h_{\text {terrain }}\left(x_{\text {tip }}, y_{\text {tip }}\right)+z_{\text {tol }} \leq z_{\text {tip }},
$$

where $z_{\text {tol }}$ is a safety tolerance of two metres. The surface terrain is interpolated using matrix Singular Value Decomposition [6], which is simple to implement and executes rapidly provided the matrix of terrain data has low rank.

The nonlinear optimal control problem given by Equations (27)-(33) are solved numerically using direct transcription: the original optimal control problem is transformed into a nonlinear programming problem (NLP). DIRECT [7], a general purpose re-usable optimal control software package was 
TABle 1: Parameters governing case study simulations

\begin{tabular}{|lc|lc|}
\hline Parameter & Value & Parameter & Value \\
\hline Payload mass $[\mathrm{kg}]$ & 150 & Aircraft mass $[\mathrm{kg}]$ & 1200 \\
Cable diameter $[\mathrm{mm}]$ & 2.5 & Aircraft Wing Area $\left[\mathrm{m}^{2}\right]$ & 20 \\
Payload area $\left[\mathrm{m}^{2}\right]$ & 0.75 & Aircraft profile drag constant & 0.015 \\
Payload drag constant & 1 & Aircraft lift curve slope & 5.2 \\
Initial tow speed $[\mathrm{m} / \mathrm{s}]$ & 50 & Max engine thrust $[\mathrm{N}]$ & 10000 \\
Initial cable length $[\mathrm{m}]$ & 600 & Cable density $\left[\mathrm{kg} / \mathrm{m}^{3}\right]$ & 7850 \\
\hline
\end{tabular}

employed. DIRECT uses the sparse nonlinear solver SNOPT to solve NLPs, implemented in Matlab 7 via mex files. The Hermite-Simpson discretization method was chosen as it enabled rapid convergence for the initial guess and scenario considered. Constraint Jacobians were estimated using finite differencing. The initial guess was the system equilibrium configuration with no control input.

\section{Results}

Results are presented from a representative case study demonstrating remote payload transportation using a cable towed beneath an aircraft. The key system parameters are listed in Table 1 . The aircraft initially has the coordinates $(-2000,-200,500)$ and a heading $\chi=10^{\circ}$ and angle of attack $\alpha=4.73^{\circ}$. The coordinates of the rendezvous location on the ground are $(-1000,0,1.5)$. Figures $3-5$ show the results of the simulation. Along with the raw optimization results obtained using DIRECT, the continuous solution for the states, obtained by integrating the state equations using the control found from DIRECT, are also presented. Close agreement between the two suggests a feasible optimization solution. To enhance clarity, the surface terrain is not shown in Figures 3 and 4. See in the results that for successful rendezvous with minimum applied control, the aircraft performs a series of shallow dives 


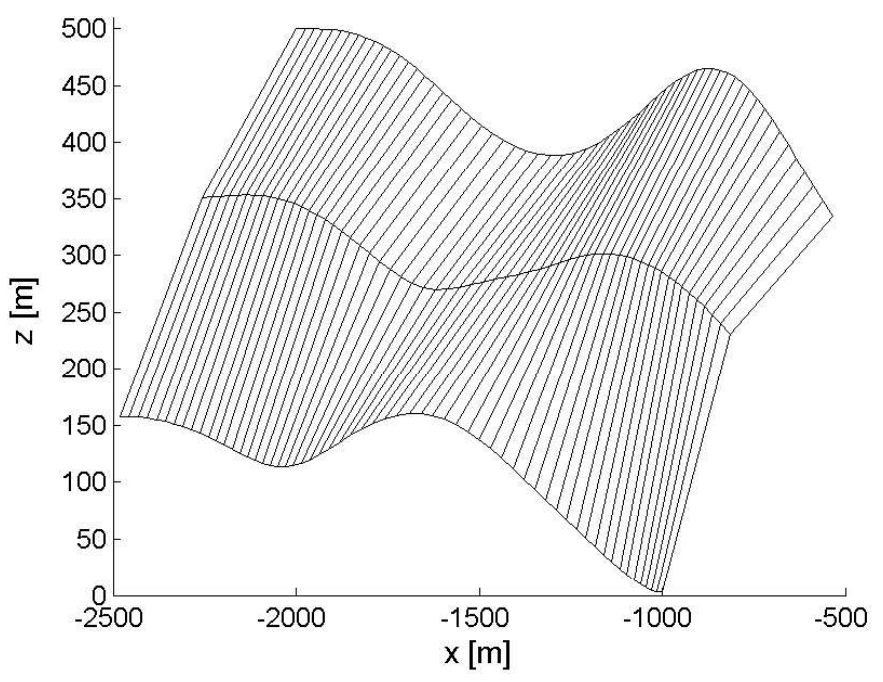

Figure 3: Cable configuration in $x$ - $z$ plane

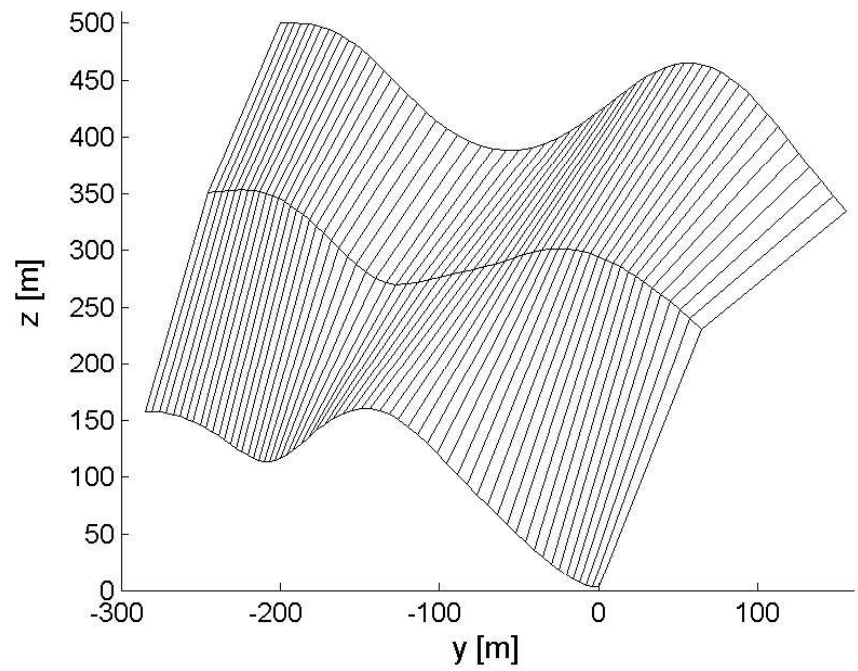

Figure 4: Cable configuration in $y$ - $z$ plane 

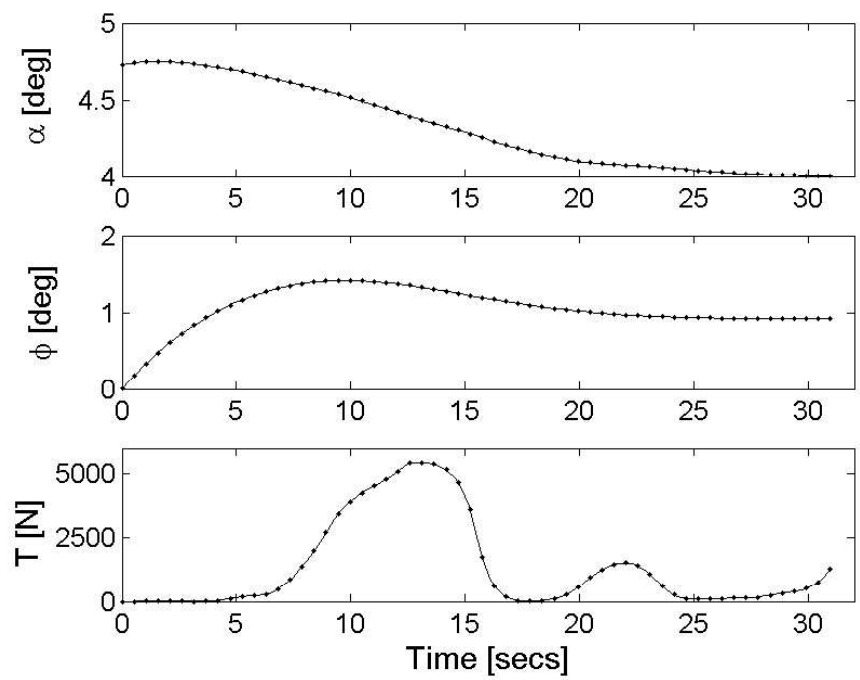

FIgURE 5: Aircraft control: ( $\cdots)$ DIRECT solution, $(-)$ propagated solution and pull-ups, while the cable is slowly deployed. For safe operation, aircraft control constraints may be required, since low aircraft thrust levels are experienced during the rendezvous manoeuvre.

\section{Conclusions}

The concept of using a flexible cable towed beneath an aircraft to transport payloads without landing was successfully demonstrated using a guidance algorithm designed via nonlinear optimal control. This optimal control problem was solved using direct transcription. Three-dimensional dynamic coupling was preserved between the aircraft dynamics and the motion of the cable and payload. The cable tip motion was indirectly controlled by aircraft maneuvering and prescribing the reel acceleration of the cable. Numerical results were presented for a typical payload transportation operation. 
Acknowledgements: This work is supported by the School of Aerospace, Mechanical and Manufacturing Engineering, RMIT University.

\section{References}

[1] Y. Jun, K. Hall, A. Bennett and P. Bridges. Optimal guidance for airborne cable pickup system. AIAA Paper 84-1893, 1984, pages 379-384. C232

[2] P. Trivailo, D. Sgarioto and C. Blanksby. Optimal control of aerial tethers for payload rendezvous. Proceedings of the 5th Asian Control Conference, Melbourne, Jul. 20-23, 2004, pages 285-294. C232

[3] D. Sgarioto and P. Trivailo. Cable assisted rendezvous for aircraft with surface locations. Proceedings of the $16^{\text {th }}$ International Federation of Automatic Control World Congress, Prague, 4-8 Jul. 2005, pages 1-6. $\mathrm{C} 232$

[4] P. Williams, D. Sgarioto and P. Trivailo. Optimal control of an aircraft-towed flexible cable system. Proceedings of the 11th Australian International Aerospace Congress, Melbourne, 13-17 Mar. 2005, pages 1-21. C232

[5] P. Williams, D. Sgarioto and P. Trivailo. Motion planning for an aerial-towed cable system. AIAA Guidance, Navigation, and Control Conference and Exhibit, San Francisco, Aug. 15-18, 2005, pages 1-31. C232, C236, C237

[6] A. Long and C. Long. Surface approximation and interpolation via matrix SVD. The College Mathematics Journal, 32(1):20-25, 2001. $\mathrm{C} 239$ 
[7] P. Williams. User's Guide to DIRECT Version 1.15. Technical Report, Melbourne, Australia, Nov. 2004. C239 